\title{
Analysis and Research on the Application of Sampling Big Data Statistical Method
}

\author{
Yan Liu \\ Xi'an Eurasia University, Xi'an, Shaanxi 710065, China
}

\begin{abstract}
Under the current statistical environment and technical conditions, there is a certain lag in the publication of statistical data. This means that there is a time lag in the completion of reports, which may delay the judgment of the current economic situation. Network real-time analysis based on big data analysis has gradually become the main force of data analysis. This paper puts forward the basic idea of understanding the statistical inference problem of non-probabilistic sampling. Sampling methods can consider sample selection based on sample matching, link tracking sampling method, etc., so that the obtained non-probabilistic samples are like probabilistic samples, so the statistical inference theory of probabilistic samples can be adopted. Random sampling technology and non-random sampling technology still have many applicable scenes, which are not only the scenes of traditional sampling survey in the past, but also applied to more modern information scenes with the times.
\end{abstract}

Keywords: Big Data; Sampling Method; Application.

\section{Introduction}

With the rapid development and wide application of big data and artificial intelligence technology, the data in the whole world is showing a spurt of growth. Big data technology has made obvious application achievements in medicine, science and technology, marketing and other fields, and the economy and society have begun to enter the era of big data [1]. The statistical characteristics in the era of big data challenge the traditional sampling analysis and question the representativeness and reliability of the sampling analysis results.

The basic idea of sampling method is to randomly extract sub-samples from the initial big data instead of the original data to estimate, predict and statistically infer the model. The difficulty of sampling method lies in designing the probability distribution of sub-samples. The simplest probability distribution is uniform distribution, and a large number of research documents show that the probability distribution using statistical leverage value as sub-samples is better than uniform distribution [2-3]. At present, some commonly used software tools have been unable to collect, manage, process and analyze these data in an effective time [4]. Therefore, we must provide a brandnew method to obtain effective information, adapt it to the large-scale data processing mode, transform the obtained simple data information into rich and diverse information assets with high growth rate, and improve the process optimization ability and decision-making power.

\section{Impact of Big Data Technology Development on Traditional Statistical Methods}

\subsection{Impact of Collection Method}

In the aspect of data collection and processing, the current government statistical work mainly focuses on calculating and analyzing structured data, and various statistical and investigation methods are mainly formulated for structured data. However, there is no mature investigation and analysis method for information data such as pictures, audio and video, and it is still a new research field to refine information data into mature statistical data products to serve the society.

Therefore, it is difficult to accurately estimate the subpopulation with selected random samples. At present, it can only be estimated by constructing various estimators and statistical models [5]. For big data, there will be no such problem. After all, the data accumulated through different ways already 
exists and has not been screened, which not only has a high data dimension, but also has a high recording frequency. It is very unlikely that there will be insufficient data when subdividing massive data sets from any dimension.

\subsection{Impact of Statistical Authority}

In the era of big data, the free sharing of Internet data makes the public become participants and users of data, and the government statistics department is no longer the authority of social data. The analysis and release of data are also expanded from government to enterprise behavior, and the inconsistency of published index data will inevitably lead to the influence of government statistical authority, and reduce the public's recognition of government statistical data.

\subsection{Impact of Statistical Data Security}

In the past, when the information industry was not developed, people wanted to know all the information of a certain thing or group, and they could only obtain data through census and interview. The work was cumbersome and required high manpower and material costs, and the data information obtained was not $100 \%$ satisfactory. It can be said that the appearance of sample survey solved these difficulties to a great extent.

At present, most of the original statistical data are reported by the reporting enterprises to the statistical departments through the online direct reporting platform, and various statistical indicators are updated and released through the websites of the statistical bureaus at all levels. The statistical reporting system needs fixed IP and real-name registration system operation, which leaves a potential safety hazard for network hackers to steal user information. Network hackers can learn about users' business information from user data and collect users' personal preferences and behavior privacy, thus the security of statistical data is fully challenged.

\section{Sampling Method}

\subsection{Tracking Sampling Method}

The definition of this method is that the subsequent sample units are selected from the units related to the previous sample units and tracked according to this interrelated relationship network, to find other sample units of the target population and expand the sample.

$r$ random samples are taken, the observed value of samples is $\left\{y_{i}^{*}, X_{i}^{*}\right\}_{i=1}^{r}$, and the corresponding sampling probability is $\left\{\pi_{i}^{*}\right\}_{i=1}^{r}$. Then the weighted estimation based on sampling samples is:

$$
\tilde{\beta}=\arg \min _{\beta}\left(y^{*}-X^{*} \beta\right)^{T} W\left(y^{*}-X^{*} \beta\right)
$$

The weight matrix $W=\operatorname{diag}\left(\left\{\pi_{i}^{*}\right\}_{i=1}^{r}\right)$.

The results show that $\tilde{\beta}$ is unbiased. In particular, if the sampling probability ignores the differences of different sub-sample distributions, they are all set to uniform distribution, that is, $\pi_{i}=1 / n, i=1,2, \Lambda, n$. The estimated quantity is:

$$
\tilde{\beta}_{U N I F}=\arg \min _{\beta}\left\|y^{*}-X^{*} \beta\right\|^{2}
$$

$\widetilde{\beta}_{U N I F}$ is called consistency estimation. This consistency estimation algorithm has the advantages of less computation time and unbiased, but the disadvantage is that the variance of the estimator is too large. 
Its procedure is a snowball sampling process. First, select some interviewees from the target group (also called seeds or round 0), and investigate these interviewees. Then, ask the interviewees to invite $2 \sim 4$ other members of the target group (round 1) to participate in the survey, and so on.

Follow-up respondents were also asked to invite other members $(2 \sim 4)$ to participate in the survey. In this way, the sample is getting bigger and bigger like snowballing. This process continues until the sample reaches a certain "equilibrium", that is, the final sample composition is stable and has nothing to do with the number and type of seeds. Under certain conditions, the observation sample obtained by this sampling method is equivalent to a probability sample.

Adaptive sampling is to use the density estimation of samples in the first stage to determine the sampling area in the second stage with different ratios, to obtain more sample units. Indirect sampling starts with a probability sample that is not in the target population, and then connects the target population with the sample unit to calculate the selection probability of the target population.

\subsection{Estimation of Optimal Sampling Design for Linear Model}

Generalized models are widely used in many disciplines, including business, computer science, education, genetics and others [6]. The unknown parameter $\eta$ can usually be estimated by maximum likelihood estimation (MLE) and maximizing its log likelihood function, namely:

$$
\eta_{M L E}=\arg \max _{\eta} l(\eta ; X, Y)=\arg \max _{\eta} \sum_{I=1}^{N}\left[\left(-y_{i} x_{i}^{T} \eta\right)+\varphi\left(x_{i}^{T} \eta\right)\right]
$$

Actually, there is no general approximation form to get the maximum likelihood estimate $\hat{\eta}_{M L E}$, and numerical calculation is often used in the iterative process. A commonly used iterative process is Newton's method. For generalized linear models, Newton's iterative method is applicable to the following formulas until $\hat{\eta}^{(t+1)}$ converges.

$$
\begin{aligned}
& \hat{\eta}^{(t+1)}=\hat{\eta}^{(t)}-\left(\frac{\partial^{2} l(\hat{\eta})}{\partial \hat{\eta} \partial \hat{\eta}^{T}}\right)^{-1} \frac{\partial l\left(\hat{\eta}^{(t)}\right)}{\partial \eta} \\
& =\hat{\eta}^{(t)}-\left(X^{t} W X\right)^{-1} \frac{\partial l\left(\hat{\eta}^{(t)}\right)}{\partial \eta} \\
& =\hat{\eta}^{(t)}-\left\{\sum_{i=1}^{n} W_{i}\left(\hat{\eta}^{(t)}\right) x_{i} x_{i}^{T}\right\}^{-1} \frac{\partial l\left(\hat{\eta}^{(t)}\right)}{\partial \eta}
\end{aligned}
$$

Because it takes $O\left(n d^{2}\right)$ computation time in each iteration, and the optimization process takes $O\left(\zeta n d^{2}\right)$ time, where $\zeta$ is the number of iterations required for convergence of the optimization process. This interesting example is its large sample size.

For such a large sample problem, the calculation time $O\left(n d^{2}\right)$ of a single run may be too long, so let's use iterative method to calculate it. Therefore, calculation is a bottleneck in the application of generalized linear model for large-scale data.

Due to the limited computing resources, the commonly used statistical methods have some limitations. The commonly used method to extract useful information from data is sub-sampling method, which uses the estimated value based on sub-samples randomly selected from complete data to approximate the estimated value of complete data. Literature [7] puts forward an effective subsampling method for unbalanced data sets under local case control, in which the method is realized by balancing sub-samples.

Personality characteristics of optimal subsampling. Most researches on subsampling algorithms (under the background of linear regression) focus on algorithm problems. One exception is the research in Literature [8]. In this process, the estimators and estimators of estimation are expressed approximately from the lever of algorithm. However, when these approximations remain unchanged, there is no accurate theoretical study. In this paper, we strictly prove that the result estimators of 
general subsampling algorithm are consistent with MLE of complete data, and establish the asymptotic normality of the result estimators.

\section{Application Analysis of Statistical Method of Sampling Big Data}

Random sampling makes the results more stable and accurate, and eliminates many non-random noises. In this paper, the user multi-dimensional credit data of a company is used, and the combination forecasting model of classification decision tree is selected to predict the overdue repayment risk of user loans, to illustrate the importance of random samples to the data analysis results.

For the data needed for forecasting, we can use the personal information data, bank record data and historical behavior data provided by the capital demander, but these data come from different systems, and there will be some noise more or less, which interferes with the correctness of the analysis results, and it takes time and effort to check them one by one. Therefore, we need to find suitable methods to solve this problem.

The multi-dimensional credit data of users used in this paper includes three aspects, one is the basic attributes of users (user ID, gender, occupation, education level, marital status and account type), the other is the credit card bill records (credit card amount, bill balance, circulating interest and cash advance), and the third is the overdue behavior of users.

In the original data set, there are 50536 individual data, and after desensitization of data and elimination of individuals with missing values of any variable, there are 48631 remaining. In the growth process of the decision tree, the training sample sets are grouped continuously, and each branch of the decision tree is allowed to grow by grouping, until all the groupings are no longer meaningful, the growth process of the decision tree ends (see Figure 1).

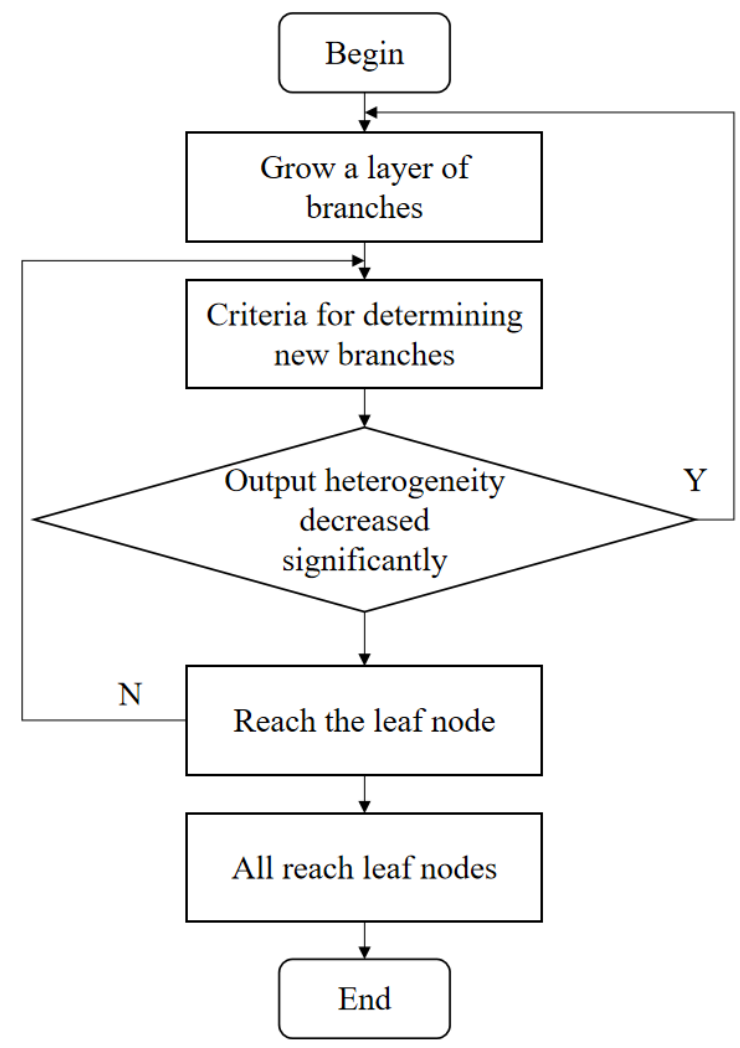

Figure 1. Decision tree growth process diagram

The complexity is set to increase from 0 , the minimum sample size of the node is 20 , the first four candidate variables of the current best grouping variable are selected, the maximum depth of the tree is 30 , and bootstrap sampling is carried out for the combined classification tree for 20 times. The 
confusion matrices of the prediction results of the two types of prediction models on the test sample set are shown in Table 1 and Table 2 respectively.

Table 1. Prediction result of single classification tree

\begin{tabular}{|c|c|c|c|c|}
\hline \multirow{2}{*}{} & \multicolumn{2}{|c|}{ Training set } & \multicolumn{2}{c|}{ Test set } \\
\cline { 2 - 5 } & 0 & 1 & 0 & 1 \\
\hline 0 & 30217 & 336 & 12087 & 345 \\
\hline 1 & 3301 & 524 & 2018 & 88 \\
\hline
\end{tabular}

Table 2. Combined classification tree prediction results

\begin{tabular}{|c|c|c|c|c|}
\hline \multirow{2}{*}{} & \multicolumn{2}{|c|}{ Training set } & \multicolumn{2}{c|}{ Test set } \\
\cline { 2 - 5 } & 0 & 1 & 0 & 1 \\
\hline 0 & 32367 & 38714 & 13018 & 2044 \\
\hline 1 & 3 & 66 & 5 & 9 \\
\hline
\end{tabular}

It can be seen from the table that although the performance of single classification tree is slightly better than that of combined classification tree on the training sample set, for new samples, the combined prediction model established by bootstrap samples is obviously better than that of single prediction model, which proves that the combined prediction model established on multiple groups of random samples can improve the robustness of prediction results to a certain extent, thus reflecting that random samples still have certain application value in the process of big data analysis.

\section{Summary}

In this paper, aiming at the statistical inference of non-probabilistic sampling under the background of big data, the statistical inference theory of probabilistic samples can be used for inference. In the aspect of weight construction and adjustment, we can consider the methods of weight construction and adjustment based on pseudo-design, weight construction and adjustment based on model, and weight construction and adjustment based on tendency score to obtain basic weights like probability samples. On the whole, although the research results of big data statistical analysis are still in the initial stage, only some preliminary explorations have been made at present. However, the research literature surrounding the characteristics of mass, high speed and real-time online update has provided a basic research framework for big data statistical analysis, and laid an important foundation for more valuable research to emerge.

\section{Acknowledgments}

Subject: The 13th Five-Year Plan of Education science in Shaanxi Province.

Project name: Research on the transformation and development of economic Statistics major under the background of big data. Project Number: SGH20Y1490.

\section{References}

[1] Wan Shuchen, Jin Yongjin. Research on the Application of Multiple Sampling Frames in Small and Micro Enterprises under the Background of Big Data [J]. 2021(2018-9):126-140.

[2] Li Shaotai, Liu Jianping. Study on the sampling method of CPI representative items compiled by big data [J]. Statistics and Information Forum, 2020, 35(3):10.

[3] Idy. Probe into the sample survey of government economic statistics in the era of big data [J]. China Business Review, 2020, 000(006): P.231-232.

[4] Gao zhanqing, Liu yixuan, he Jianfeng. discussion on the method of multiple sampling frames under the background of big data [J]. statistics and decision, 2020(1):5-9. 
[5] Li Yi, Mi Zichuan. Uniform sampling design and numerical analysis of big data mining [J]. 2021(20154): 3-6.

[6] Huang Philosophy, He Yulin, Wei Chenghao, et al. Random sample partition model of big data and related analysis and calculation technology [J]. Data Acquisition and Processing, 2019, v.34; No. 155(03):5-17.

[7] Lv jiran. application of big data statistical analysis method in economic management field [J]. journal of consumption, 2020, 000(007):145-146.

[8] Ren Liying. Sampling Survey Method in American Political Studies [J]. American Studies, 2020(3):83106. 